\title{
Cosine Modulated Non-Uniform Filter Bank with Improved Computational Efficiency
}

\author{
*Jyotsna Ogale is with Department of Electronics and Communication Engineering, Samrat Ashok Technological \\ Institute, Vidisha (M.P.), India.,E-mail jyoti.ogale@yahoo.com \\ \#Alok Jain Department of Electronics and Instrumentation Engineering, Samrat Ashok Technological Institute, \\ Vidisha (M.P.), India., E-mail:alokjain6@ rediffmail.com
}

\begin{abstract}
In this correspondence, a simple design method for multirate near perfect reconstruction (NPR) integer decimated filter bank with non-uniform frequency spacing and linear phase property, that involves optimization of only single parameter, is proposed. It is derived from the uniform cosine modulated filter bank (CMFB) by merging some relevant band pass filters. The design procedure and the structure of the uniform CMFB are mostly preserved in the non-uniform implementation. The parent filter of the filter bank is formulated as an interpolated finite impulse response (IFIR) filter. The IFIR digital filters permit efficient hardware implementations due to less number of multiplier coefficients. Design examples show that the proposed approach provides good performance with less computational complexity at the cost of slight increase in system delay.
\end{abstract}

Index Terms - Cosine modulation, non-uniform filter bank, near perfect reconstruction.

\section{Introduction}

Multirate filter banks find wide applications in many areas of digital signal processing such as sub-band coding, transmultiplexer, image, video and audio compression, adaptive signal processing [1-3]. On the basis of time-frequency resolution, filter bank can be classified in two categories, which include, uniform and non-uniform filter bank. The uniform filter bank provides fixed and uniform time frequency decomposition [1], whereas, in some applications like audio analysis and coding, broadband array signal processing non-uniform and variable time-frequency resolution may lead to better performance and reduced arithmetic complexity, which is provided by nonuniform filter bank (NUFB) [4-6]. Meaning thereby, efficient structure and design procedures for NUFB are highly desirable. Over the years, a number of design methods have been proposed by different authors [610]. Among these, only few of them possess linear phase (LP) property. The tree structure method [1] is an easy way to design LP-NUFB via cascading uniform filter bank. However, the limitation of decimation factors and the long system delay are two major drawbacks of this technique. The resulting filters tend to be long, since they are convolutions of the interpolated filters. Most of the available approaches [6-13] for NUFBs use standard constrained or unconstrained optimization techniques to obtain the design, which tend to be computationally expensive, when high order filters are used. In wideband audio signal analysis and coding, filter banks with high stop band attenuation greater than $100 \mathrm{~dB}$ is required. Moreover, it is difficult to design NUFBs with high stop band attenuation and LP property. In [11], a simple design method for NUFBs is proposed. It is based on the design of a uniform cosine modulated filter bank and is applicable only to non-uniform integer-decimated filter banks. Moreover, it still involves complicated nonlinear optimization with large number of parameters. In 2006, Zhong [21] has proposed new design method in NUFB. During 2007, Zing et al. [12] proposed interpolated FIR prototype filter to design the NUFB.

A simple design approach for linear phase NUFB is presented here. The approach is based on the merging of uniform CMFBs therefore, the design of non uniform CMFB is also reduced to the design of the prototype filter. With this approach NUFBs with high

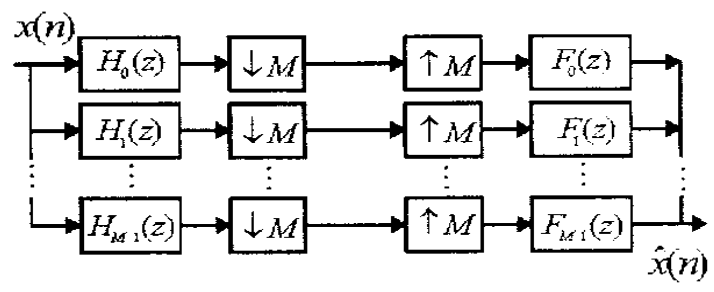

Fig.1 $M$-channel uniform filter bank

stop band attenuation up to $110 \mathrm{~dB}$ can be easily designed. A single variable optimization is used to obtain minimum value of amplitude $\left(E_{\max }\right)$ and aliasing $\left(E_{a}\right)$ distortions. This paper is organized as follows: The narrow band filter design technique is briefly reviewed in section 2 . The design problem is stated in section 3 together with closed form expressions for analysis/synthesis filters in uniform/non uniform filter bank. In section 4, optimization algorithm is discussed. Section 5 provides some design simulations along with discussions. Finally in section 6 some concluding remarks are given.

\section{Narrow band filter design}

In this section, a narrowband low pass FIR filter is designed using Kaiser window function. The order of the filter is given by: 


$$
N=\frac{-20 \log _{10}\left(\sqrt{\delta_{p} \delta_{s}}\right)-13}{14.6\left(\frac{\omega_{s}-\omega_{p}}{2 \pi}\right)}+1
$$

Where $\delta_{p}$ and $\delta_{s}$ are the maximum pass band and stop band ripples, and $\omega_{p}$ and $\omega_{s}$ are the pass band and stop band edge frequencies. With the same design parameters the interpolated FIR filter is described by the equation:

$B(z)=F\left(z^{L}\right) G(z$

$F\left(\mathrm{z}^{L}\right)$ has a periodic frequency response with period $2 \pi$ $/ L$ is called the up sampled version of the model filter and $G(\mathrm{z})$ is called the image suppressor filter designed to attenuate the extra unwanted pass bands of up sampled filter below the specified maximum stop band level. The overall realization is called interpolated finite impulse response filter.

As outlined in [14], the process of designing an IFIR filter, is as follows:

2.1) Select a suitable stretch factor $L$. Reduction in computation by IFIR method depends upon the $L$. As $L$ increases the order of the model filter decreases. Consequently, a higher order image suppressor filter is needed to remove the unwanted replicas. The optimum value of $L$ is given as [15] :

$L_{\mathrm{opt}}=\left[\frac{2 \pi}{\omega_{p}+\omega_{s}+\sqrt{2 \pi\left(\omega_{s}-\omega_{p}\right)}}\right]$

2.2) Design model filter $F(z)$.Using the same specifications for pass band and stop band ripple as of FIR, a model filter is designed with pass band and stop band edge are:

$$
\begin{gathered}
\omega_{p m}=L\left(\omega_{p}\right) \\
\omega_{s m}=L\left(\omega_{s}\right)=\frac{L}{M} \pi
\end{gathered}
$$

2.3) Up sample model filter $F(z)$ by $L$ to create $F\left(z^{L}\right)$. In the time domain model filter is up sampled by the factor $L$ by inserting $L-1$ zero valued coefficients between each sample. The frequency response becomes periodic.

2.4) Design the image suppressor filter $G(z)$ to remove replicas. Image filter is designed with pass band $\omega_{p s}$ and stop band $\omega_{s s}$ edge at $\left(\frac{\pi}{2 M}\right)$ and $(2 \pi / L-\pi / M)$ respectively and convolving these two filters in the time domain final IFIR filter results.

\section{Cosine modulated filter bank}

\subsection{Uniform}

Cosine modulation is a cost effective technique for $M$ band filter bank [1]. In this approach all the filters of analysis and synthesis section are obtained by cosine modulation of single linear phase prototype low pass filter which normally has linear phase and a finite length impulse response as shown in Fig. 1

The impulse responses of filters of analysis and synthesis sections for uniform CMFB are obtained from the closed form expressions as given by [1]:

$$
\begin{aligned}
& h_{k}(n)=2 b(n) \cos \left[(2 k+1) \frac{\pi}{2 M}\left(n-\frac{N}{2}\right)+(-1) \frac{\pi}{4}\right] \\
& f_{k}(n)=2 b(n) \cos \left[(2 k+1) \frac{\pi}{2 M}\left(n-\frac{N}{2}\right)-(-1) k \frac{\pi}{4}\right] \\
& \text { for } 0 \leq k \leq M-1,0 \leq n \leq N-1
\end{aligned}
$$

\subsection{Construction of Non-uniform Filter Banks}

In the $M$ - channel uniform division filter banks, the basic frequency range $-\pi \sim+\pi(\mathrm{rad} / \mathrm{s})$ is divided into a number of bands with equal width of $2 \pi / M$. Here, $M$ is the number of parallel filters and the independent number of pass bands on the basic frequency range. A real coefficients filter bank has a symmetric band with respect to the origin. Therefore, the positive frequency range is also divided into $M$ components of equal bandwidth and decimation is done by the maximum factor of $M$. In non-uniform case, basic frequency range is divided into a number of non-uniform bands and the value of decimation factors are not the same. In the case concept of cosine modulating low pass filters is applied [11,12,13]. After designing the required uniform CMFB, the corresponding NUFB is obtained by merging the relevant band pass filters of analysis and ${ }_{3}$ synthesis section of the uniform filter bank as described below [11].

We define $\bar{H}_{i}(z), \mathrm{i}=0,1,2 \ldots . \bar{M}-1$, to be the filters obtained by merging the $l_{i}(\geq 1)$ adjacent analysis filters, i.e., $H_{k}(z)$ 's, for $\mathrm{i}=n_{i}$ through $\left(n_{i}+l_{i}-1\right)$ in a uniform $M$-channel CMFB. More specifically,

$$
\bar{H}_{i}(z)=\sum_{k=n_{i}}^{n_{i}+l_{i}-1} H_{k}(z) \quad 0 \leq i \leq \bar{M}-1
$$

where $n_{i+1}=n_{i}+l_{i}$.We define $\bar{F}_{i}(z), \quad \mathrm{i}=$ $0,1,2 \ldots . \bar{M}-1$, in a similar manner for the synthesis filters $F_{k}(z)$ 's of the $M$-channel CMFB. That is,

$$
\bar{F}_{i}(z)=\frac{1}{l_{i}} \sum_{k=n_{i}}^{n_{i}+l_{i}-1} F_{k}(z) \quad 0 \leq i \leq \bar{M}-1
$$

Then $\bar{H}_{i}(z)$ and $\bar{F}_{i}(z), \mathrm{i}=0,1,2 \ldots \ldots \bar{M}-1$, form a new set of analysis and synthesis filters in the $\bar{M}$-channel non-uniform CMFB. Here, we assign the $\bar{M}$ number of integer set $l_{i}(\mathrm{i}=0,1,2 \ldots . \bar{M}-1)$ to each band on the positive frequency range from 0 to $\pi$. And we define the following $L$ 
$L=\sum_{k=0}^{\bar{M}-1} l_{k}$

Then, the bandwidth of the analysis filters becomes

$B W_{i}=\frac{2 \pi l_{i}}{L}(\mathrm{i}=0,1,2 \ldots . \bar{M}-1)$.

It may be noted that $n_{0}=0<n_{1}<n_{2}<\ldots<n_{M}=\bar{M}$, and $l_{0}+l_{1}+l_{2}+\ldots . .+l_{M-1}=\bar{M}$. Fig. 2 shows the resulting overall structure of the $\bar{M}$-channel nonuniform CMFB.

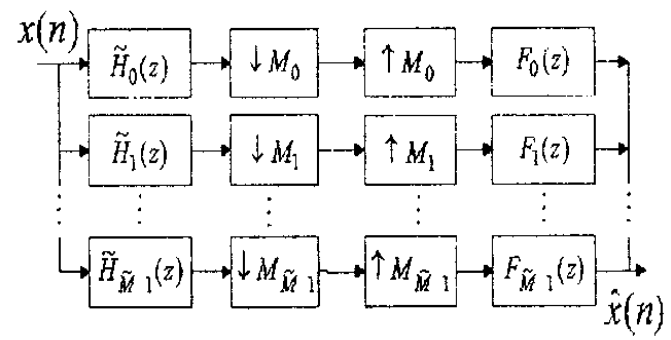

Fig.2 $\tilde{M}$-channel nonuniform filter bank

In the figure, $M_{i}\left(L / l_{i}\right), \mathrm{i}=0,1,2 \ldots . \bar{M}-1$, amounts to the decimation and interpolation ratio for the $i$ th channel. When $l_{i}$ is the divisor of $L$, the decimation factor becomes an integer.

The centre frequency of the analysis filters from 0 to $\pi$ is written by

$$
\begin{gathered}
\omega_{c_{i+1}}=\omega_{c_{i}}+\left(B W_{i}+B W_{i+1}\right) / 4(\mathrm{i}=0,1,2 \ldots . \bar{M}-3) . \\
\text { Where } \omega_{c 0}=0, \omega_{c_{i}}=B W_{0} / 2+B W_{1} / 4 \text { and } \\
\omega_{c_{M}-1}=\pi .
\end{gathered}
$$

And, we have a restriction on the center frequency. An integer $p$ must satisfy

$$
\omega_{c i}=\frac{\pi}{2 M_{i}}(2 p+1) \quad(\mathrm{i}=0,1,2 \ldots \ldots \bar{M}-2) .
$$

Where $p=1 \sim M_{i}-2$. The change of parameter $p$ indicates the possible equal space shift of the center frequency of the band width of which is $2 \pi / M_{i}$. The occurrence of large aliasing caused by the existence of the positive and negative frequency components will be avoided by the above condition.

The design procedure for a non-uniform filter bank with integer decimation factors is as follows:

1) Obtain $\bar{M}$, which is the $l \mathrm{~cm}$ of decimation factors.

2) Design a cosine modulated NPR $M$ - channel uniform filter bank according to (4).

3) Merge the relevant band pass filters of the uniform section to obtain the analysis and synthesis filters $\bar{H}_{i}(z)$ and $\bar{F}_{i}(z)$ of the non-uniform bank according to $(7-8)$.

4) If the set of given integer decimation factors make a feasible partition, do not make any changes to the structure.
5) If the set represents non-feasible partition, the structure as given in [11] should be used to implement the same. The modified structure shifts the frequency band of such a channel to a suitable band

\section{Optimization technique}

In NPR, perfect reconstruction condition is relaxed by allowing a small amount of distortion. Three types of distortions occur at the reconstructed output, i.e, amplitude $\left(E_{\max }\right)$, phase and aliasing $\left(E_{a}\right)$ [1]. The aliasing and phase distortion can be eliminated by careful design of the linear phase FIR filter. However, amplitude distortion can not be eliminated completely but can be minimized by applying optimization technique. Initially; Johnston [17] developed a nonlinear optimization technique. Later on many prominent authors such as Creusere et al [18], Lin et al [19], Jain et al [16], have simplified it using linear optimization technique with objective function as given below:.

$\phi=\left.\max || B\left(e^{j \omega}\right)\right|^{2}+\mid B\left(\left.e^{j(\omega-\pi / M)}\right|^{2}-1 \mid\right.$

(11)

for $0 \leq \omega \leq \pi / M$

In this work, same objective function in modified form is used for the design of non-uniform filter bank, as given below vaidynathan [1]:

$\phi=\left.\max \left|\sum_{i=0}^{\bar{M}-1}\right| \bar{B}_{i}\left(e^{j \omega}\right)\right|^{2}-1 \mid$,

for $0 \leq \omega \leq \pi / \bar{M}$

where, $\bar{M}$ is number of channels in non-uniform filter bank and $\bar{H}_{i}\left(e^{j^{\circ}}\right)$ is the frequency responses of the filters of the non-uniform section.

The optimization routine for designing non-uniform CMFB is summarized as follows:

(i) Choose the initial value of sampling frequency $\left(F_{s}\right)$,number of bands ( $M$ ), stop band attenuation $A_{s}$ and Pass band ripple and stretch factor $(L)$.

(ii) Initialize the Normalized pass band $\left(\omega_{p}\right)$ and stop band $\left(\omega_{s}\right)$ frequency.

(iii) Selected pass band frequency $\omega_{p m}=L \omega_{p}$ and stop band frequency $\omega_{s m}=L \omega_{s}$ for the model filter.

(iv) Calculate the cut-off frequency $\left(\omega_{c m}\right)$ transition band and Filter length $\left(N_{m}\right)$ of model filter.

(v) Design model filter.

(vi) Design up-sampled filter by up-sampling the impulse response coefficients model filter 
by a strech factor $L$.

(vii) Select $\frac{\omega_{p m}}{L}$ and $\left(2 \pi / L-\omega_{s}\right)$ as a pass band and stop band frequencies for image suppressor filter.

(viii) Calculate the cut-off frequency $\left(\omega_{c s}\right)$, transition band and Filter length $\left(N_{s}\right)$ of image suppressor filter.

(ix) Design the image suppressor filter.

(x) Obtain sub filters of analysis and synthesis Sections of uniform filter bank using cosine modulation scheme.

(xi) Merge the relevant band pass filters of uniform section to obtain sub filters of non-uniform section

(xii) Choose the step size (step) and its search direction (dir) is equal to either 1 or -1 .

(xiii) Compute $\omega_{c m}=\omega_{c m}+$ dir $\times$ step and objective function using (12).

(xiv). When difference between previous iteration and current iteration becomes negligible the optimization process is halted. Otherwise, it returns to (xii).

(xv) Return to (xiv) if the current objective function is smaller than the previous one. Otherwise, step $=$ step $/ 2, \operatorname{dir}=-\operatorname{dir}$ and return to (xiii).

(xvi). Calculate aliasing distortion.

\section{Design examples}

In this section, 5- channel and 3- channel NUFBs are designed and the performance of proposed technique is compared with the earlier reported work $[5,11,12,20]$.

5.1. This example is quoted to compare the performance with recent work of Zing et al. [12]. In the work of [12], 5-channel NUFB with integer decimation factors $(4,4,8,8,4)$ is designed using IFIR based prototype filter. In this example, 5-band NUFB is designed with the same specifications as in [12]: Stop band attenuation $A_{s}=110 \mathrm{~dB}$. The band edge frequencies are:

$\omega_{1}=\pi / 4, \omega_{2}=\pi / 2, \omega_{3}=5 \pi / 8, \omega_{4}=3 \pi / 4$. In this case, $n_{0}=0 . n_{1}=2, n_{2}=4, n_{3}=5, n_{4}=6, n_{5}=8$; and $l_{0}$ $=2, l_{1}=2, l_{2}=1, l_{3}=1, l_{4}=2$. The obtained overall filter length in this case is 103 with $L=2$. The magnitude responses of prototype filter bank and distortion parameter are shown in Fig. (3-6). The obtained value of peak deviation from unity of the distortion parameter is $0.0059 \mathrm{~dB}$ and aliasing distortion is $3.93 \times 10^{-11}$ as shown in Table $\mathbf{1}$, where performance is compared with latest work of [12].

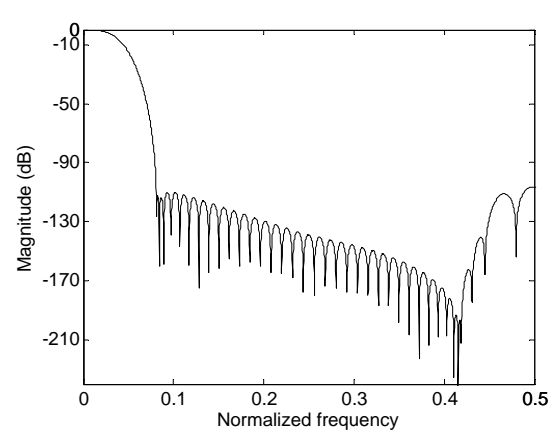

Fig. 3 Magnitude response of optimized prototype filter

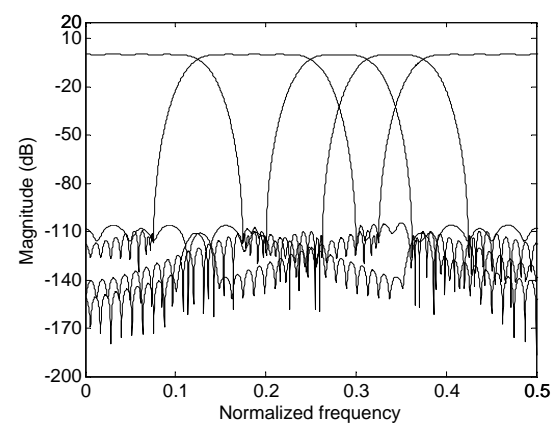

Fig. 4 Magnitude response of five-channel filter bank.

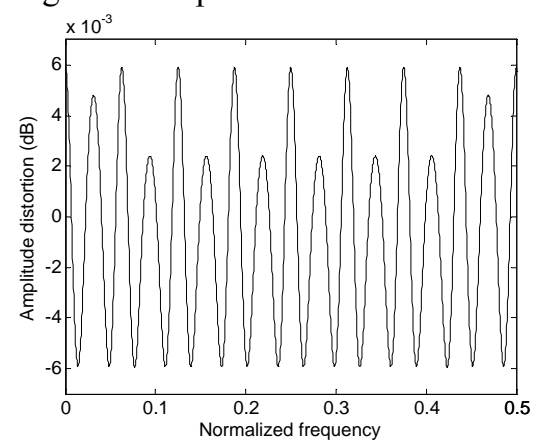

Fig. 5 Amplitude distortion plot.

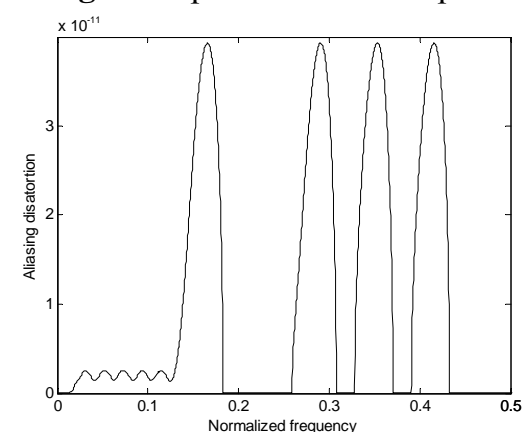

Fig. 6 Aliasing distortion plot.

5.2. This example considers the design of a 3-channel non-uniform filter bank with decimation factors $(4,4$, $2)$. The filter bank has a feasible partition. The $1 \mathrm{~cm}$ of $(4,4,2)$ is $M=4$. The stop band attenuation $\left(A_{s}\right)$ is kept at $110 \mathrm{~dB}$, and interpolation factor $L=2$. A four channel uniform Pseudo-QMF bank with model filter length $N_{m}$ $=27$ and suppressor filter length $N_{s}=21$ is first designed. The overall filter length is given by $N=$ $L N_{m}+N_{s}$ and is equal to 75 . The analysis/synthesis filters of the non-uniform filter bank are constructed from the analysis/synthesis filters of the uniform filter 
bank. In this case $l_{0}=1, l_{1}=1, l_{2}=2$ and $n_{0}=0$, $n_{1}=1, n_{2}=2$. The band edge frequencies are $\omega_{1}=\pi / 4, \omega_{2}=\pi / 2$. The magnitude responses of the optimized prototype filter, the corresponding analysis filters of the NUFB, optimized value of amplitude distortion function are plotted in Fig. (7-9). It is observed that for the designed filter bank maximum amplitude distortion is $E_{\max }=3.77 \times 10^{-3}$ and aliasing distortion is $E_{a a}=1.14 \times 10^{-11}$. Table 2 provides a performance comparison between the proposed method and the methods in [5], [11], [20]. It is quite oblivious that proposed method offers much better performance than the method in [5] and [11]. As compared to the method [20], our performance is very much comparable. Compared to all, proposed design is computationally more efficient. Designed non-uniform filter bank provides better performance with less computational complexity.

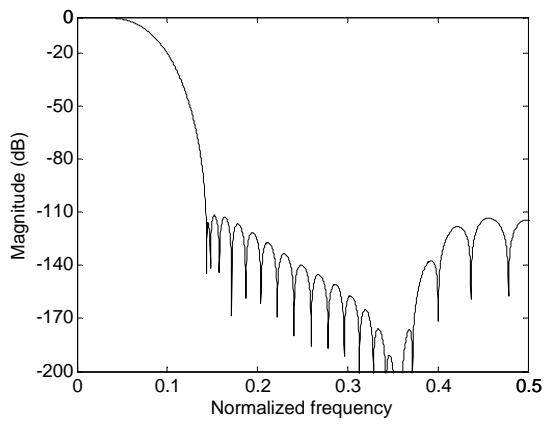

Fig. 7 Magnitude response of optimized prototype filter

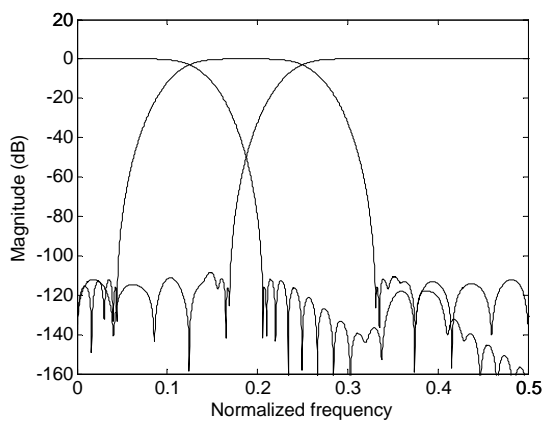

Fig. 8 Magnitude response of three- channel filter bank

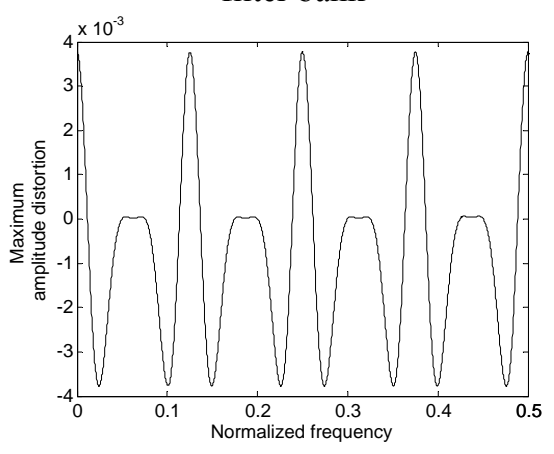

Fig. 9 Amplitude distortion plot.
5.3. The third example is also a 3-channel NUFB, but of different decimation factors. In this case decimation factors are $(2,6,3)$. Prototype filter with specification stop band attenuation $\left(A_{s}\right)=110 \mathrm{~dB}$, interpolation factor $L=2$ has been designed. The model and suppressor filter lengths are $N_{m}=51$ and $N_{s}=19$ respectively. In this case, non-uniform filter bank has been designed from six-channel uniform filter bank with, $l_{0}=3, l_{1}=1$, $l_{2}=2$, and $n_{0}=0, n_{1}=3, n_{2}=4$. The band edge frequencies are $\omega_{1}=\pi / 2, \omega_{2}=2 \pi / 3$. The obtained value of maximum amplitude distortion is $E_{\max }=$ $3.02 \times 10^{-3}$ and aliasing distortion is less than $E_{a a}=$ $2.67 \times 10^{-11}$. The magnitude responses of prototype filter, NUFB, optimized value of amplitude distortion are shown in Fig. (10-12). Table 2 provides a comparative performance between the proposed method and the methods in [5].

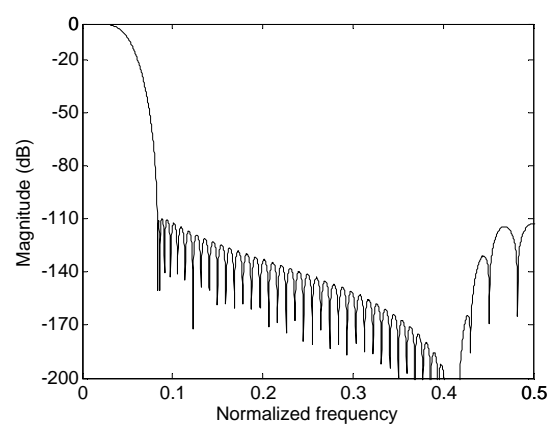

Fig. 10 Magnitude response of optimized prototype filter

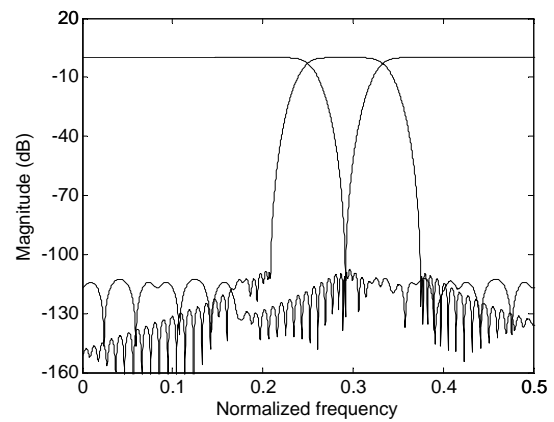

Fig. 11 Magnitude response of three- channel

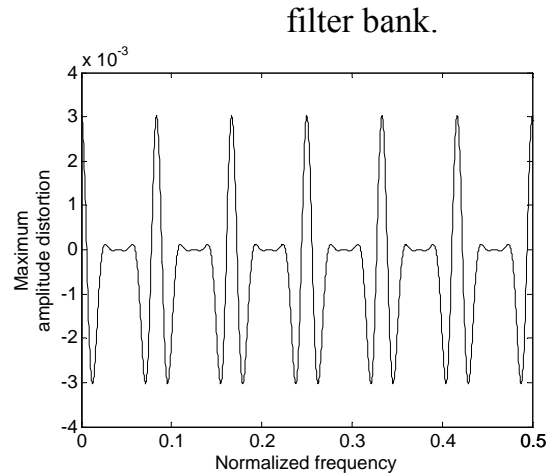

Fig. 12 Amplitude distortion plot.

Case studies are presented to demonstrate the effectiveness of the design. 3 and 5 channel symmetric non-uniform filter banks were designed and the 
amplitude characteristics of analysis filters are shown in Fig. 3, Fig. 7 and Fig. 10. The positive frequency range is clearly divided into 3 and 5 non-uniform bands. These filter banks have integer decimation factors with feasible partition. For the designs the Kaiser windowed LPF were used as initial filters for minimization of the performance function. Further, as a tool for optimization, the gradient algorithm was utilized. In cost effective design for the filter banks, IFIR approach is one of the techniques, which provides significant reduction in computational cost. In the proposed work same technique is introduced to provide the reduction in computation and improvement in the performance of the filter bank. It has been observed that the proposed method over existing one offer much better performance with reduction in computational complexity. As shown in Fig.13. For all the three cases computational requirements in proposed method is minimum. Performance comparisons with previous publications [5,11,12,19] given in Table (1-3) show that apart from lower values of distortion parameters, the proposed method provides $32.43 \%, 26.53 \%$ and $13.88 \%$ reduction in computation for three and five channel filter banks.

On one hand, comparisons with Tree-Structure NUFBs show that the Tree-Structure can either be PR or NPR depending on the FBs used in the design. Whereas, on the other hand, proposed method has limitation that it can only design NPR FBs. The method is efficient but do not achieve perfect reconstruction. The advantage of our method is that it can be used to design a feasible or non-feasible partition NUFB with good performance. (All the Tables and Fig. 13 are appended at the last).

\section{Conclusion}

A simple and computationally efficient design of NUFB is presented. In traditional design approaches, it is difficult to design the NUFB at high stop band attenuation above $100 \mathrm{~dB}$. The proposed work eliminates this constraint by exploiting the design process of cosine modulation and obtained NUFB with a feasible partition property. The prototype filter is formulated as an interpolated FIR filter and is indirectly designed by varying the pass band edge of a low-order model filter. This method is suitable particularly for large number of channels where high order filters with unequal pass bands have to be designed with small distortion and aliasing. The proposed method is efficient and simple and guarantees high stop band attenuation while keeping the distortion and aliasing small. Such filter banks are needed in a wide variety of applications like speech coding and speech enhancement. The proposed technique is useful in variety of applications of audio and video field.

\section{REFERENCES}

[1] P.P.Vaidyanathan, Multirate Systems and Filterbanks, Prentice-Hall, Englewood Cliffs, NJ, 1993.
[2] Q.G. Liu, B. Champagne, D.K.C. Ho, "Simple design of over sampled uniform DFT filterbanks with application to sub-band acoustic echo cancellation", IEEE Trans. on Signal Processing, .80(5), (2000), pp. 831-847.

[3] G.Gu., E.F. Badran, "Optimal design for channel equalization via the filterbank approach", IEEE Trans. on Signal Processing,52(2), (2004), pp. 536-544.

[4] G.Shi, X.Xie and W.Zhong, "Recent advances and new design method in nonuniform filterbanks", Proc. of IEEE Int. Conf. on Communications, Circuits and Systems, vol.1, (May 2006), pp. 211-215.

[5] X.M. Xie, X.Y. Chen and G, M, Shi,"A simple design method of linear-phase nonuniform filterbanks with integer decimation factors", Proc. of Int. Symposium on Circuit and Systems, vol. 1,(Aug. 2006), pp. 724-727.

[6] X.M. Xie, S. C. Chan and T. I. Yuk, "A class of perfect reconstruction nonuniform cosinemodulated filter-bank with dynamic recombination", Proc. of XI European Signal Processing Conf., Toulouse, France, vol. 2, (Sept. 2002), pp. 549-552.,

[7] S. C. Chan and X. M. Xie, "A rational subdivision scheme using cosine-modulated wavelets", Proc. of Int. Symposium on Circuits and Systems, Vancouver, Canada, vol. 3, (May 2004), pp. 409-412.

[8] S. C. Chan, X. M. Xie and T. I. Yuk, "Theory and design of a class of cosine-modulated nonuniform filterbanks", Proc of IEEE Int. Conf. on Acoustics Speech, and Signal Processing, Istanbul, Turkey, vol.1,(June 2000), pp.504-507.

[9] E. Elias, P.Lowenborg, H. Johansson and L.Wanhammar, "Tree-structured IIR/FIR octave-band filterbanks with very lowcomplexity analysis filters", IEEE Int. Symp. on Circuits and Systems (ISCAS), vol.2, (May 2001), pp. 533-536.

[10] O. A. Niamut and R. Heusdens, "Sub-band merging in cosine-modulated filterbanks", IEEE Signal Processing letter, 10(4), (Apr. 2003), pp. 111- 114.

[11] J.Li, T.Q.Nguyen and S.Tantaratana, “A simple design method for near perfect reconstruction non-uniform filterbanks", IEEE Trans. on signal processing, 45(8), Aug. 1997.

[12] Z. Zing and Y. Yun,"A simple design method for nonuniform cosine modulated filterbank", IEEE Int. Symp. On Microwave, Antenna, Propagation and EMC Tech., (2007), pp.10521055 ,

[13] J. Lee and B.G. Lee, "A design of nonuniform cosine modulated filterbanks", IEEE Transactions on Circuits and System-II, 42(1), November 1995. 
[14] Y.Neuvo, C.Y.Dong and S.K.Mitra,"Interpolated Finite Impulse Response Filters", IEEE Transactions on Acoustic, Speech, Signal Processing, vol.ASSP-32, pp. 563-570, June 1984.

[15] A.Mehrina and J.A.Willson, "On Optimal IFIR Filter Design", IEEE Proceedings of the 2004 International Symposium on Circuits and System, vol.3,pp. 133-136,May 2004.

[16] A Jain, R Saxena and S.C. Saxena, "An improved and simplified design of cosine modulated pseudo QMF filterbanks", Digital Signal Processing, vol.16,no.3, pp. 225-232, 2006.

[17] J.D Johnston, "A filter family designed for use in quadrature mirror filterbanks", Proc. IEEE Int. conf. Acoustics , Speech and Signal Processing, Denver, pp. 291-294,1980.
[18] C.D Cresure, S.K Mitra, "A simple method for designing high-quality prototype filters for M-band pseudo QMF banks", Trans. on Signal Processing, vol.43, pp. 1005-1007, 1995.

[19] Y.P Lin, P.P Vaidyanathan, "A Kaiser window approach for the design of prototype filters of cosine-modulated filterbanks", IEEE Signal

Processing Letter, vol.5, no.6, pp. 132134,1998. [20] R K Soni, A. Jain, "An Optimized Design of Non-uniform filterbank using Blackman window family", International Journal of Signal and Image Processing, vol.1,no.1, pp. 18-23,2010.

[21] G. Shi, X. Xie, X.Chen and Wei Zhong. , "Recent Advances and New Design Method in Nonuniform Filter Banks", IEEE Trans. on Signal Processing, (2006), pp. 211-215.

Table 1. Performance comparison with earlier reported works for five-channel NUFB

\begin{tabular}{|c|c|c|c|c|c|c|c|c|c|}
\hline Work & $\begin{array}{c}\text { Channels } \\
\text { /Decimation } \\
\text { Factors }\end{array}$ & $\begin{array}{l}\text { Technique } \\
\text { used }\end{array}$ & $A_{s}$ & $L$ & $N$ & $\begin{array}{l}\text { er len } \\
N_{m}\end{array}$ & $N_{s}$ & $\begin{array}{l}\text { Amplitude } \\
\text { distortion }^{\mathrm{a}}\end{array}$ & $\begin{array}{l}\text { Aliasing } \\
\text { Distortion }\end{array}$ \\
\hline $\begin{array}{l}\text { Lee } \text { et al [13] } \\
\quad(1995)\end{array}$ & $\begin{array}{c}\text { Five channels } \\
(4,4,8,8,4)\end{array}$ & $\begin{array}{c}\text { Cosine } \\
\text { modulation } \\
\text { (FIR) }\end{array}$ & $46.3 \mathrm{~dB}$ & - & 41 & - & - & $0.027 \mathrm{~dB}$ & - \\
\hline $\begin{array}{l}\text { Zijing et al [12 ] } \\
\text { (2007) }\end{array}$ & $\begin{array}{l}\text { Five channels } \\
(4,4,8,8,4)\end{array}$ & $\begin{array}{c}\text { Cosine } \\
\text { modulation } \\
\text { (IFIR) }\end{array}$ & $110 \mathrm{~dB}$ & 4 & 163 & 31 & 39 & $0.0068 \mathrm{~dB}$ & $3.37 \times 10^{-11}$ \\
\hline Proposed & $\begin{array}{c}\text { Five channels } \\
(4,4,8,8,4)\end{array}$ & $\begin{array}{c}\text { Cosine } \\
\text { modulation } \\
\text { (IFIR) }\end{array}$ & $110 \mathrm{~dB}$ & 2 & 103 & 43 & 17 & $0.0059 \mathrm{~dB}$ & $3.93 \times 10^{-11}$ \\
\hline
\end{tabular}

Table 2. Performance comparison with earlier reported works for three-channel NUFB.

\begin{tabular}{|c|c|c|c|c|c|c|c|c|c|c|}
\hline S.N & Work & Channels & $\begin{array}{l}\text { Decim- } \\
\text { ation } \\
\text { Factors }\end{array}$ & $\begin{array}{l}\text { Techniq } \\
\text {-ue } \\
\text { used }\end{array}$ & $A_{s}$ & $L$ & \multicolumn{3}{|c|}{ Filter length } & $\begin{array}{l}\text { Amplitude } \\
\text { Distortion }\end{array}$ \\
\hline & $\begin{array}{l}\text { Li et al[11] } \\
\quad(1997)\end{array}$ & Three & $(4,4,2)$ & $\begin{array}{c}\text { Cosine } \\
\text { modulation } \\
\text { (FIR) }\end{array}$ & $60 \mathrm{~dB}$ & - & 65 & - & - & $7.803 \times 10^{-3}$ \\
\hline & $\begin{array}{l}\text { Xie et al[5] } \\
(2006)\end{array}$ & Three & $(4,4,2)$ & $\begin{array}{l}\text { Recomb- } \\
\text { ination } \\
\text { (FIR) }\end{array}$ & $110 \mathrm{~dB}$ & - & 63 & - & - & $7.803 \times 10^{-3}$ \\
\hline 1. & Proposed & Three & $(4,4,2)$ & $\begin{array}{c}\text { Cosine } \\
\text { modulation } \\
\text { (IFIR) }\end{array}$ & $110 \mathrm{~dB}$ & 2 & 75 & 27 & 21 & $3.77 \times 10^{-3}$ \\
\hline & $\begin{array}{l}\text { Xie } e t \text { al[5] } \\
\quad(2006)\end{array}$ & Three & $(2,6,3)$ & $\begin{array}{l}\text { Recomb- } \\
\text { ination } \\
\text { (FIR) }\end{array}$ & $110 \mathrm{~dB}$ & - & 95 & - & - & $6.631 \times 10^{-3}$ \\
\hline 2. & Proposed & Three & $(2,6,3)$ & $\begin{array}{c}\text { Cosine } \\
\text { modulation } \\
\text { (IFIR) }\end{array}$ & $110 \mathrm{~dB}$ & 2 & 121 & 51 & 19 & $3.02 \times 10^{-3}$ \\
\hline
\end{tabular}


Table 3. Computation comparisons

\begin{tabular}{cccccc}
\hline S.No & Work & Channels & Multipliers & Adders & $\begin{array}{c}\text { \% Reduction in } \\
\text { Computation }\end{array}$ \\
\hline & & & & & \\
1. & Proposed & Three & 37 & 63 & \\
& Three & 25 & 48 & $34.21 \%$ \\
2. & Proposed & Three & 49 & 95 & \\
& {$[12]$} & Five & 36 & 70 & $26.53 \%$ \\
3. & Proposed & Five & 36 & 70 & $13.88 \%$ \\
\hline
\end{tabular}

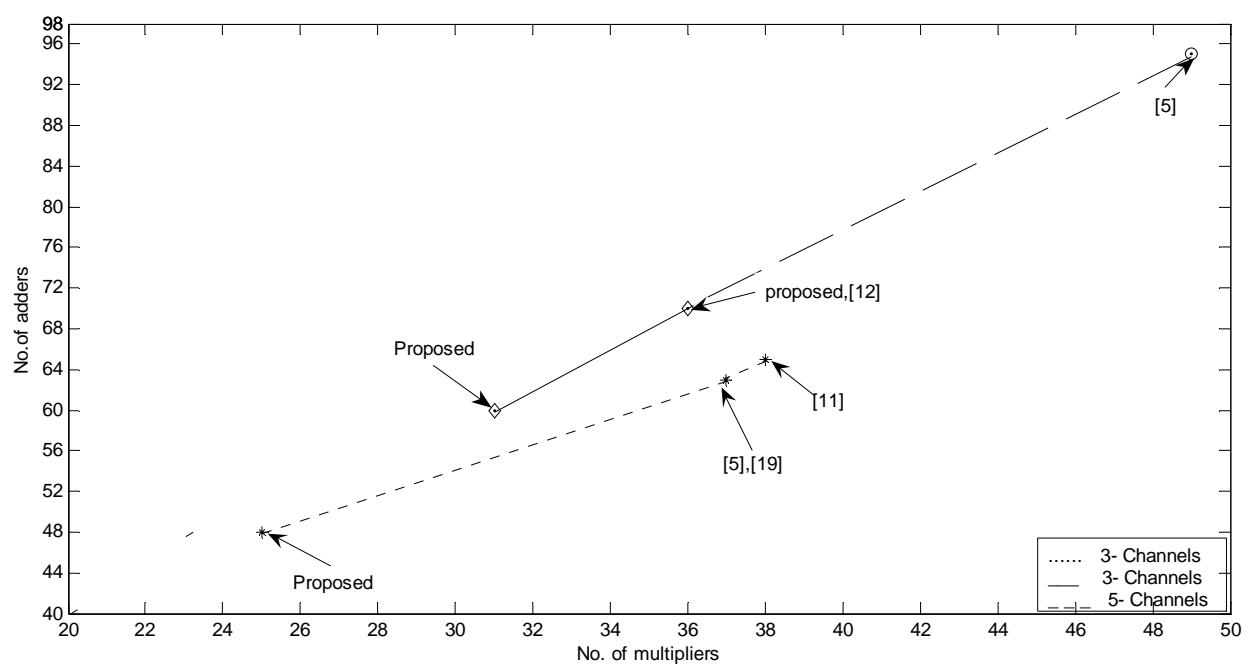

Fig.13. Computational requirement comparison between proposed and reported works.

Jyotsna v. Ogale was born in Jabalpur, Madhya Pradesh, in 1972. She persuaded the B.E and M.E degrees in Electronics and Telecommunication \& Communication System from the Rani Durgawati Vishwavidhayalaya, Jabalpur (M.P), India, in 1995 and 1998, respectively. She joined the Department of Electronics in Samrat Ashok Technological Institute, Vidisha (M.P.), in 1999 and is currently Associate Professor in EC Department pursuing Doctorate form Rajiv Gandhi Technical University (M.P), India. Her research interests include filter and multirate system
Prof. Alok Jain was born in India, in 1966. He received his B.E. (Electronics and Instrumentation) in 1988, M.Tech. (Computer Science and Technology) in 1992 and Ph.D. in 2006. He joined as a lecturer in Samrat Ashok Technology Institute (Engineering college), Vidisha. Presently, he is working as Professor and Head, Department of Electronics and Instrumentation Engineering. He has published over 30 papers in refereed journals and conference proceedings. $\mathrm{He}$ is an author of the book "Power Electronics and Its Applications," from Penram Int. Publishing (India), His current research interest includes Digital Signal Processing, filter banks, and their applications in power electronics and biomedical engineering. 